\title{
Editorial
}

\section{Slow to change: New cord clamping policies for premature infants}

\author{
N.N. Finer ${ }^{\mathrm{a}, *}$ and A. Katheria ${ }^{\mathrm{b}}$ \\ ${ }^{a}$ Department of Pediatrics, Division of Neonatology, The University California - San Diego, San Diego, CA, USA \\ ${ }^{\mathrm{b}}$ Department of Neonatology, Sharp Mary Birch Hospital for Women and Newborns, San Diego, CA, USA
}

Reviewed 4 February 2014

Revised 12 February 2014

Accepted 12 February 2014

Recently, the American College of Obstetricians \& Gynecologists (ACOG) recommended a 30-60 second delay in umbilical cord clamping for all preterm deliveries [1]. Delayed cord clamping in preterm infants has been associated with a greater than $50 \%$ reduction in intraventricular hemorrhage and less need for blood transfusions as demonstrated in a recent Cochrane meta-analysis of 10 randomized controlled trials of delayed cord clamping compared to immediate cord clamping [2]. The AAP has endorsed the ACOG statement and the Neonatal Resuscitation Program has also recommended that cord clamping should be delayed for at least 1 minute $[1,3,4]$. These statements should change the standard of care for all preterm deliveries by eliminating immediate cord clamping (ICC). A recent survey conducted of US obstetricians, however, demonstrated that only $12 \%$ of responders had an umbilical cord clamping policy [5]. The most frequent response for why there was no policy for optimal timing of umbilical cord clamping, regardless of gestational age, was "don't know." The potential for neonatal red blood cell transfusion was the only reason cited for performing a delay of cord clamping, while

${ }^{*}$ Corresponding author: Dr. Neil N. Finer, 402 Dickinson St, MPF1-140, San Diego, CA 92103, USA. E-mail: nfiner@ucsd.edu. the risk of delaying neonatal resuscitation was listed as the reason to clamp the cord immediately [5].

Historically, delayed cord clamping was common practice for cord management. In 1796 Erasmus Darwin expressed that, "Another thing very injurious to the child, is the tying and cutting the navel-string too soon; which should always be left till the child has not only repeatedly breathed, but till all pulsation in the cord ceases. As otherwise the child is much weaker than it ought to be; a part of the blood being left in the placenta, which ought to have been in the child." However, in the early 20th century this began to change with the emergence of the formal discipline of Obstetrics. Later, as neonatologists began attending deliveries, obstetricians cut the cord immediately for preterm infants to give them rapid access to the infants. For neonatologists, the goal of establishing ventilation and adequate transition in the first minute of life has resulted in near-universal adoption of immediate cord clamping, despite an absence of solid evidence that this intervention confers a benefit to babies, and increasing evidence of harm [6].

As indicated by the survey, the most common reason for obstetricians not to perform a delay in umbilical cord clamping is the risk of delaying neonatal resuscitation. The most important determinant of a healthy 
extra-uterine transition is a post-delivery increasing heart rate. An increasing heart rate is a surrogate marker for improving pulmonary blood flow. Bhatt (2013) demonstrated an acute decrease in heart rate and an acute increase in carotid artery pressure with immediate cord clamping in a preterm lamb model [7]. These parameters recovered slowly after onset of ventilation [7]. Conversely, when ventilation was initiated before clamping of the umbilical cord, there was substantially less variability in carotid artery pressures, no drop in heart rate and a progressive increase in pulmonary blood flow during the first 4 minutes. They concluded that ventilation before cord clamping in this animal model assures a smoother circulatory transition compared to immediate cord clamping.

Interestingly, while delayed cord clamping has reduced overall IVH in the largest published metaanalysis there has not been a significant reduction in severe (grade 3 or 4) IVH [2]. One possibility is that the infants most at risk for IVH, those with respiratory depression at birth, are actively excluded from receiving delayed cord clamping because of the initiation of aggressive resuscitation practices. In fact, studies using composite score of early neonatal well-being (i.e. SNAP and CRIB score) which take a composite of APGAR scores, and the amount of resuscitation needed have consistently demonstrated that the higher the composite score, the increased likelihood of death or IVH [8-10]. It is quite possible that the infants most at risk of developing IVH are not receiving the most optimal interventions.

An alternative to delayed cord clamping is umbilical cord milking, in which the unclamped umbilical cord is milked before it is clamped. However, the number of randomized controlled trials in preterm infants is small [11-13]. A study performed by our group in infants $<32$ weeks demonstrated that compared to immediate cord clamping, umbilical cord milking improved early neonatal hemodynamics and decreased the need for blood transfusions, days on oxygen therapy, and oxygen requirements at 36 weeks corrected age [14]. Recently, a pilot study of 75 extremely premature neonates ( $<29$ weeks) randomized to cord milking or immediate cord clamping demonstrated a $50 \%$ reduction in total IVH. It is possible that umbilical cord milking may prevent IVH in smaller and more immature neonates [13]. One study $(n=58)$ that compared umbilical cord milking to delayed cord clamping demonstrated no difference between the groups with respect to IVH or need for blood transfusion [15].
More data will soon be available as several randomized controlled trials are underway. The University of Oklahoma (NCT005698398 N=200 infants), the University of Rhode Island (NCT00818200, $N=212$ ) and a multicenter trial in Australia (APTS trial, currently $N=590 / 1600$ ) will all have outcomes on infants randomized to either a delay cord in clamping of at least 30 seconds compared to immediate cord clamping. Our group has recently completed a pilot trial which evaluated hemodynamics with delayed cord clamping compared to umbilical cord milking (NCT01866982). These trials will provide further evidence as to which mode of placental transfusion confers greater benefit.

Another important consideration is whether resuscitation interventions if deemed necessary can and should be administered during the placental transfusion. One company has developed a neonatal resuscitation bed (LifeStart Trolley, Inditherm, London UK) that can be placed beside the mother during delivery, and provides warming and equipment for the administration of ventilation to the newly born infant while the cord is intact [16]. One study has suggested that depressed premature infants (Apgars $<4$ ) have less placental transfusion (as judged by lower hematrocrit) with DCC compared to those with higher Apgar scores. (Songali et al 2014, Mercer et al 2014, both in preparation). The benefits of DCC may be augmented if the lung is recruited during the transfusion process, as has been shown by Bhatt et al. [7]. The lung can receive a significant amount of blood if the pulmonary vasculature relaxes as the lung opens.

At present up to $50 \%$ of very preterm infants are treated for hypotension following delivery, and it may well be that this hypotension reflects the decreased placental transfusion received by such infants with immediate cord clamping. Bhatt et al demonstrated a $50 \%$ drop in pulmonary blood flow due to the cessation of umbilical venous blood flow (oxygenated blood) from the placenta in fetal lambs receiving immediate cord clamping compared to delayed cord clamping [7].

Human studies have demonstrated that both delayed cord clamping and cord milking in preterm infants improves cardiac output, measures of systemic blood flow, and improves brain oxygen extraction, and during the first 30 seconds of delayed cord clamping, blood volume in the newborn increases by at least $12 \mathrm{ml} / \mathrm{kg}$ [12, 14, 17-19]. We believe that transitional support for the premature infant could be initiated during the placental transfusion to allow for a smoother postnatal hemodynamic transition while improving the infant's 
blood volume. Such an approach needs to be tested in premature human infants comparing supportive interventions during the delayed transfusion with early cord clamping.

The evidence to date is clear; there is no high-level evidence supporting immediate cord clamping, which has never been subjected to any controlled trial apart from its use for the control group in the recent trials. We urge providers to evaluate the large body of evidence supporting early placental transfusions (via delayed cord clamping or cord milking) and compare it to the lack of evidence supporting immediate cord clamping. We look forward to the information from the ongoing trials. However, while further trials regarding the type of placental transfusion, duration of delay, and the use of supportive respiratory interventions are ongoing, providers should be aware that the best evidence to date favors delayed cord clamping.

\section{Financial disclosure statement}

The authors declare no conflict of interest.

\section{References}

[1] Committee on Obstetric Practice, American College of Obstetricians and Gynecologists. Committee Opinion No.543: Timing of umbilical cord clamping after birth. Obstet Gynecol 2012;120:1522-6.

[2] Rabe H, Diaz-Rossello JL, Duley L, Dowswell T. Effect of timing of umbilical cord clamping and other strategies to influence placental transfusion at preterm birth on maternal and infant outcomes. Cochrane Database Syst Rev 2012;8:CD003248.

[3] Kattwinkel J, Perlman JM, Aziz K, Colby C, Fairchild K, Gallagher J, et al. Neonatal resuscitation: 2010 American Heart Association Guidelines for Cardiopulmonary Resuscitation and Emergency Cardiovascular Care. Pediatrics 2010;126:e1400-13.

[4] Raju, TNK. Don't rush to cut the cord. AAP Endorsed Statement. AAP News 2013;16-17.

[5] Jelin AC, Kuppermann M, Erickson K, Clyman R, Schulkin J. Obstetricians' attitudes and beliefs regarding umbilical cord clamping. The journal of maternal-fetal \& neonatal medicine: The official journal of the European Association of
Perinatal Medicine, the Federation of Asia and Oceania Perinatal Societies, the International Society of Perinatal Obstet. 2013.

[6] van Rheenen P. Delayed cord clamping and improved infant outcomes. BMJ 2011;343:d7127.

[7] Bhatt S, Alison BJ, Wallace EM, Crossley KJ, Gill AW, Kluckow M, et al. Delaying cord clamping until ventilation onset improves cardiovascular function at birth in preterm lambs. The Journal of Physiology 2013;591:2113-26.

[8] Richardson DK, Gray JE, McCormick MC, Workman K, Goldmann DA. Score for Neonatal Acute Physiology: A physiologic severity index for neonatal intensive care. Pediatrics 1993;91:617-23.

[9] Richardson DK, Phibbs CS, Gray JE, McCormick MC, Workman-Daniels K, Goldmann DA. Birth weight and illness severity: Independent predictors of neonatal mortality. Pediatrics 1993;91:969-75.

[10] Rautonen J, Makela A, Boyd H, Apajasalo M, Pohjavuori M. CRIB and SNAP: Assessing the risk of death for preterm neonates. Lancet 1994;343:1272-3.

[11] Hosono S, Mugishima H, Fujita H, Hosono A, Minato M, Okada T, et al. Umbilical cord milking reduces the need for red cell transfusions and improves neonatal adaptation in infants born at less than 29 weeks' gestation: A randomised controlled trial. Arch Dis Child Fetal Neonatal Ed 2008;93:F14-9.

[12] Takami T, Suganami Y, Sunohara D, Kondo A, Mizukaki N, Fujioka T, et al. Umbilical cord milking stabilizes cerebral oxygenation and perfusion in infants born before 29 weeks of gestation. J Pediatr 2012;161(4):742-7.

[13] March MI, Hacker MR, Parson AW, Modest AM, de Veciana M. The effects of umbilical cord milking in extremely preterm infants: A randomized controlled trial. J Perinatol 2013;33(10):763-7.

[14] Katheria AC LT, Woelkers D, Finer N. The effects of umbilical cord milking on hemodynamics and neonatal outcomes in premature neonates. Journal of Pediatrics 2014;164(5):10451050

[15] Rabe H, Jewison A, Alvarez RF, Crook D, Stilton D, Bradley $\mathrm{R}$, et al. Milking compared with delayed cord clamping to increase placental transfusion in preterm neonates: A randomized controlled trial. Obstet Gynecol 2011;117:205-11.

[16] Life Start Trolley. 2014.

[17] Meyer MP, Mildenhall L. Delayed cord clamping and blood flow in the superior vena cava in preterm infants: An observational study. Arch Dis Child Fetal Neonatal Ed 2012;97:F484-6.

[18] Sommers R, Stonestreet BS, Oh W, Laptook A, Yanowitz TD, Raker C, et al. Hemodynamic effects of delayed cord clamping in premature infants. Pediatrics 2012;129:e667-72.

[19] Aladangady N, McHugh S, Aitchison TC, Wardrop CA, Holland BM. Infants' blood volume in a controlled trial of placental transfusion at preterm delivery. Pediatrics 2006;117:93-8. 